\title{
Determination of Mica Cell Parameters by X-Ray Powder Diffractometry - A Case Study
}

\author{
By W. B. Stern \\ Geochemical Laboratory, Institute for Mineralogy and Petrography, Basel University, \\ Bernoullistrasse 30, CH-4056 Basel, Switzerland
}

\begin{abstract}
Possibilities and restrictions of least-square methods for mica cell refinement are briefly described. If diffractometric raw data are precise and accurate, and if geometrical errors are properly corrected, a cell refinement (determination of $a_{0}$, $b_{0}, c_{0}, \beta$ ) can be carried out rapidly, but the reliability of obtained data has to be evaluated carefully.
\end{abstract}

\section{Introduction}

Mica is a common rock-forming mineral; its potential chemical variation is an intensely studied petrologic topic e.g. as a clue to physical conditions of metamorphic rock genesis (Bailey, 1984; Frey et al., 1983). Because a strong interdependence of mica cell parameters and crystal chemistry is assumed, and because $\mathrm{Mg}, \mathrm{Fe} / \mathrm{Al}$ substitution in mica is believed to be connected with physical conditions (pressure, temperature) of mica formation, X-ray powder diffractometry is a generally adopted method of investigation (Guidotti and Sassi, 1986).

A critical examination of literature data (Naef and Stern, 1982) shows, however, that either certain published data seem to be erroneous, or certain critical factors controlling cell determination were disregarded thus far. Indeed, several difficulties arise at different stages of investigation:

- Grinding the mineral may induce a partial lattice destruction.

- The flaky shape of many sheet silicates causes strong orientation effects in powder XRD.

- Measurement of the Bragg angle $2 \theta$ has to be executed very carefully in terms of precision and accuracy.

- The mineral species, its crystal symmetry, and approximate cell data have to be known a priori.

- The specimen should consist of one single phase only.

A number of specific preparation techniques have been proposed in order to reduce orientation effects (Blount and Vassiliou, 1979). One common procedure combines a Guinier exposure for $a_{0}$ and $b_{0}$ determination and diffractometry for measurement of $c_{0}$. Sometimes, however, all cell constants are determined by diffractometry. Single crystal diffractometry - as a measurement method - is seldom applied, for it is time-consuming and concerns individual mica crystals only, not a bulk sample.

In 1963 a least-squares method for general cell parameter determination and indexing by XRD was proposed and was later updated (Appleman and Evans, 1973). This approach is currently used in many computer-based commercial diffractometers as a part of the integrated software package. This promising and widespread cell refinement technique has not been applied critically in mica determination; a data bank based literature search (Physical Briefs, Georef, Chemical Abstracts) did not reveal any signficiant citations for the past 10 years. Therefore, an attempt has been made to use critically the Appleman/Evans refinement technique to investigate a series of mica specimens, extracted from rocks of different origins. Details of crystallographic and chemical data will be published elsewhere.

\section{Evaluating Optimal Refinement Conditions}

Calculated powder diffraction data [e.g. taken from Borg and Smith (1969)] are particularly useful for evaluating optimal refinement conditions:

- d-values contain virtually no experimental error

- Miller indices are known and can be introduced into the refinement routine.

- The result is known; thus it is possible to control certain variables, like cell parameters and error tolerances, as starting points for refinement.

For all preliminary evaluations the same set of data was used $\left(2 \mathrm{M}_{1}\right.$ muscovite), namely

- 29 of the strongest reflections in the $2 \theta$ range of 8 to $78^{\circ}$. - 3 reflections were a priori indexed $(-114),(025),\left(\begin{array}{lll}0 & 0 & 10\end{array}\right)$.

- Crystal class, extinction conditions, angle $\beta$.

The starting values for refinement $(a, b, c)$ were chosen close to the true cell data: differences not exceeding 0.1 to $0.2 \AA$, error tolerances according to recommended values.

As can be seen from Figure 1, correct results were obtained when the starting point was quasi-identical to true data. If, however, starting points are not close $( \pm 0.1 \AA)$, true data are only found using a specific error range: either too small or too large error tolerances may lead to entirely erroneous cell parameters. An optimal error tolerance seems unpredictable; thus, even optimal raw data may produce ambiguous results.

\section{The first intrinsic source of error: Sample Geometry}

When experimental data (and not calculated d's like those in the above mentioned case) are processed, the ambiguity of the results will tend to increase. The first intrinsic source of error has to do with sample geometry: depending on grain size, surface morphology or specimen alignment, the reflecting crystal planes may be out of the X-ray optical focus. Because a slight geometrical aberration causes measurable deviations of the observed $d$-values, especially at low $\theta$, either a mechanical specimen alignment (Smith and Leider, 1968), or an individual mathematical correction has to be done for every sample measurement. The usual software routine ( $\theta$ calibration) does not help properly, for the actual geometric correction varies from one sample to the next, as routine measurements on series of samples clearly indicate. A common solution to this problem is the addition of an internal standard to the sample powder, which is then contaminated so that further examination on this subsample may be restricted. Internal standards may display either many reflections and thus provoke undesired interferences, or only a few reflections, therefore being unsuitable for the entire range of measurement because the $\Delta \mathrm{d} / \mathrm{d}$ relationship is non-linear (Figure 2a). Fortunately, sheet silicates with their dominant basal reflections allow a very simple and effective correction procedure: after a plot of all calculated c-parameters of a given specimen against their respective d's, an extrapolation towards a Bragg angle of $180^{\circ} 2 \theta$ is possible, where a geometrical error is zero (angle between primary X-ray beam and sample surface $=90^{\circ}$, angle between 


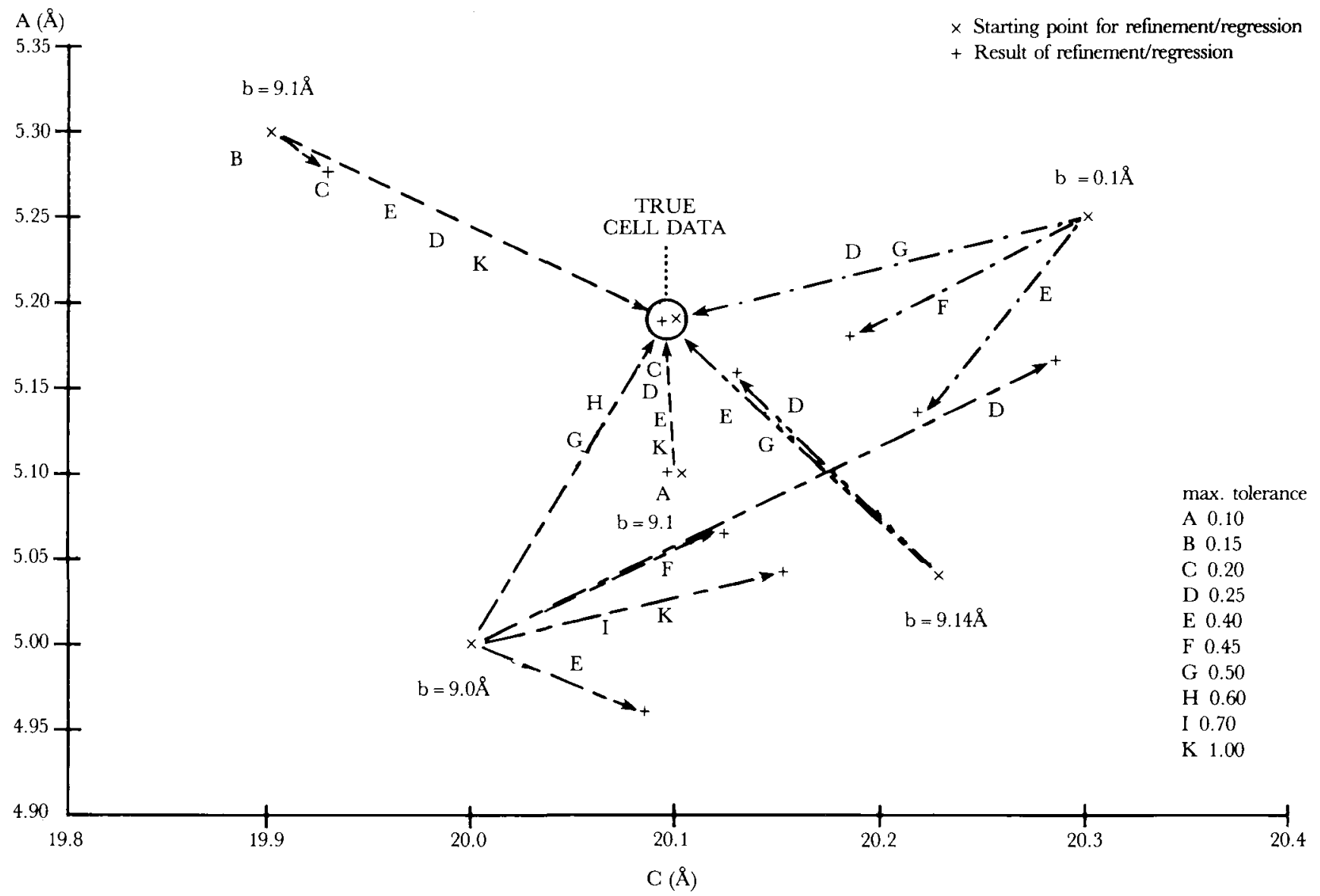

Figure 1. Least-squares refinement on recalculated powder data (2M $M_{1}$ muscovite, Borg and Smith, 1969). Same set of 29 d-values, (3 indexed) may either lead to correct cell values, or to wrong ones when error tolerances are slightly varied.

diffracted X-ray beam and sample surface $=90^{\circ}$ ). Thus, without an internal standard, and without a mechanical specimen alignment, the true $c$ for every specimen can still be found by extrapolation. Calculating back the difference between observed and true $\mathrm{d}$ for every basal reflection, $\Delta \mathrm{d}$ $(\AA)$ for the entire range of measurement can be plotted (Figure $2 \mathrm{~b}$ ), and a correction term $\Delta \mathrm{d}$ be found for every reflection. This correction term is expectedly large for low $\theta$ angles, low for high angles, and is, of course, different from one specimen to another. If the same specimen is placed at slightly different heights (ca $\pm 0.1 \mathrm{~mm}$ ) in the sample holder, the influence of sample geometry on measured d's, and the effect of the described correction procedure can be demonstrated clearly (Table 1).
The second source of error: Data Reduction

A second sounce of error is data reduction. According to details of on-line reduction routines, a weak reflection may be suppressed, or a strong one may be interpreted as superposition of several - perhaps too many - individual reflections. Every diffractogram should therefore carefully be compared with the data of the reduction routine used. At the same time, the specific mica polymorph should be determined, as well as the presence of any second mineral phase.

A manual, off-line evaluation of the diffractogram would give poor results, because a correct $\mathrm{K} \alpha_{2}$ elimination is difficult. The precision of a $2 \theta$ measurement should be in the order of $\leqslant 0.02^{\circ} 2 \theta$ which is only possible when appropriate software for data reductions is used.

Table 1.

Mica Mounted at Varying Height in Sample Holder of Diffractometer.

Sample: phengitic mica 3T, KAW 685

\begin{tabular}{|c|c|c|c|c|c|c|c|c|}
\hline $\begin{array}{l}\text { Measured } \\
\text { neflection }\end{array}$ & $\begin{array}{c}\text { D-variation } \\
\text { due to } \pm 0.02^{\circ} 2 \theta\end{array}$ & \multicolumn{6}{|c|}{$\begin{array}{l}\text { Observed peak position without d-correction } \\
\text { Setting }\end{array}$} & \\
\hline & & \multicolumn{2}{|c|}{ High } & \multicolumn{2}{|c|}{ Medium } & \multicolumn{2}{|c|}{ Low } & \\
\hline (hkl) & $(\AA)$ & $2 \theta$ & $\AA$ & $2 \theta$ & $\AA$ & $2 \theta$ & $\AA$ & \\
\hline 104 & 0.00328 & 23.12 & 3.844 & 22.88 & 3.884 & 23.09 & 3.849 & \\
\hline 108 & 0.00178 & 31.25 & 2.860 & 30.95 & 2.887 & 31.20 & 2.864 & \\
\hline \multirow[t]{2}{*}{118} & 0.00096 & 42.39 & 2.131 & 42.17 & 2.141 & 42.35 & 2.132 & \\
\hline & & \multicolumn{6}{|c|}{ Recalculated data after d-cornection } & $\bar{d}(\AA)$ \\
\hline 104 & & & 3.866 & & 3.860 & & 3.863 & $3.863 \pm 0.003$ \\
\hline 108 & & & 2.871 & & 2.876 & & 2.872 & $2.873 \quad 0.002$ \\
\hline 118 & & & 2.137 & & 2.136 & & 2.136 & 0.0003 \\
\hline
\end{tabular}




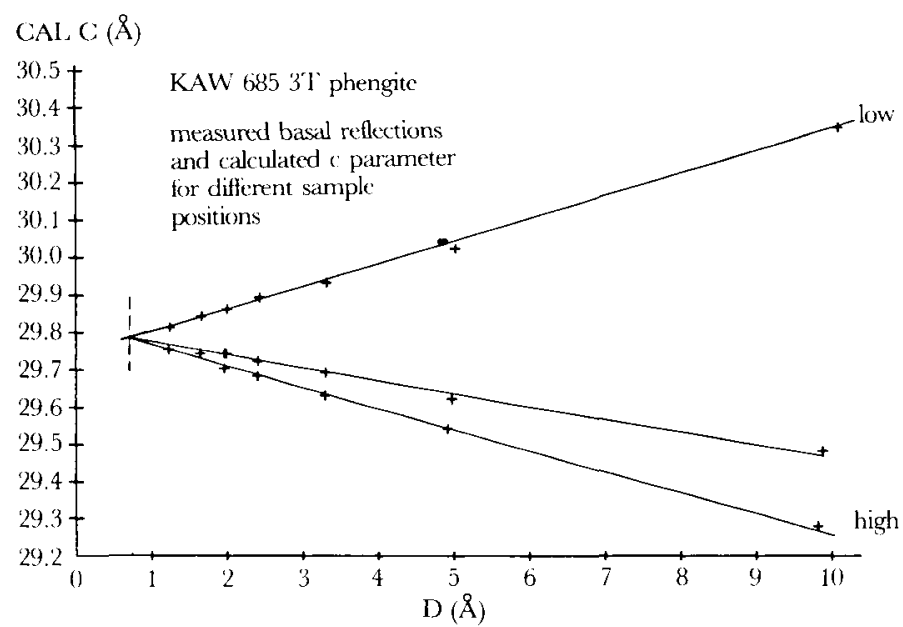

Figure 2a. Effect of sample alignment on peak position: measured basal reflections and cairulated c parameter for different sample positions. At high angles $2 \theta$ (low d-values) musurement errors due to specimen misalignment become negligibly small.

\section{The third source of error: Allowable Choice of Frogramming Parameters}

A tuird source of error is the choice of program parameters allowed by the software when performing a least-squares refinement:

- When the initial cell parameters - as a starting point for refinement - are too far off from the true parameters to be found, the result will most probably be erroneous. The number of rejected (i.e. not properly fitted) reflections will be large ( 30 to $50 \%$ or more of given d's). Because the true parameters are not known a priori, optimal settings for starting points and error tolerances are not known either.

- When certain reflections are intially indexed and one was erroneously selected or when a second (unidentified) phase is present.

- When the number and/or quality of given d's is insufficient.

\section{Evaluating Refinement Data}

Because any given set of reflections may produce different cell parameters when input parameters to the least-squares procedure are varied, an ambiguity results in how to distinguish the true cell values from incorrect ones. In order to

* The author is highly indebted to Dr. Henke of Karlsruhe Technical High School for his effort and indispensable support.

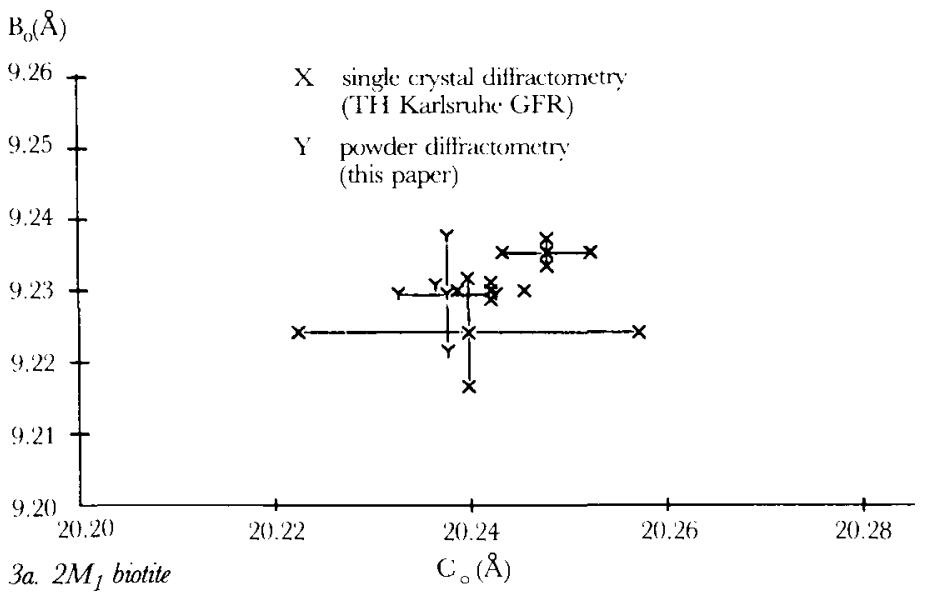

Figure 3. Comparison of poweder data and single-crystal data obtained from micas.
DELTA $\mathrm{D}(\AA)$

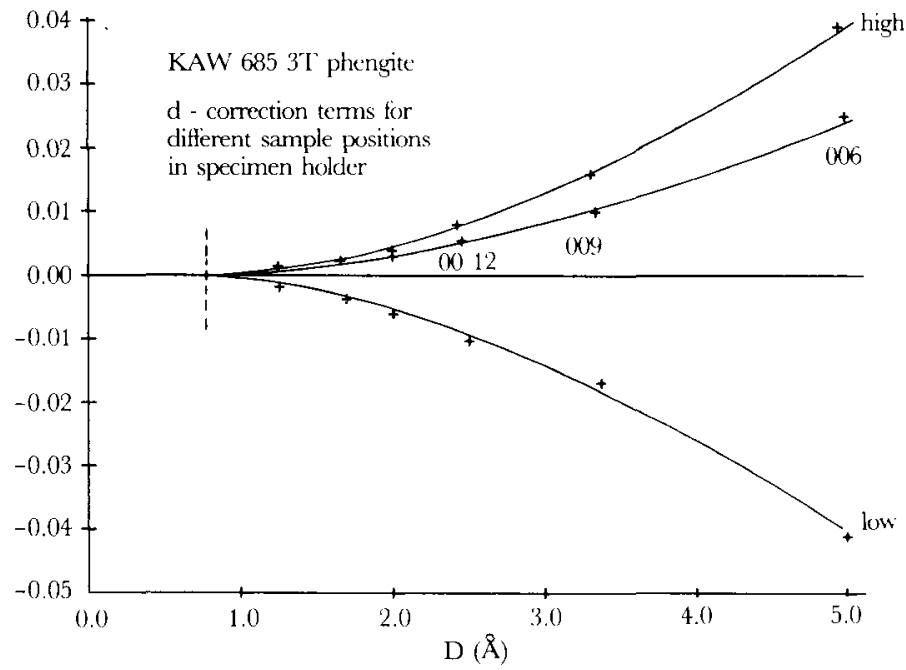

2b. Using basal reflections, the difference between measured $d$ and true $d$ can be deter mined for the entire range of measurement, and for each sampleinen.

evaluate this uncertainty, two samples consisting of several mica flakes were studied independently by single crystal diffractometry*. The results are plotted in Figure 3: singlecrystal and powder data correspond well when the respective statistical error is considered. Whether 3 or 9 reflections out of 25 to 35 are initially indexed, the result remains the same, but the number of iterations may increase, whereas the percentage of rejected (not fitted) reflections decreases. One may conclude that the smaller the difference is between results obtained on 3 preset $h \mathrm{~h} \ell \mathrm{s}$ vs 9 preset $\mathrm{hk} \ell \mathrm{s}$, the better the final results are.

A second, but weaker criterion for evaluating refinement data is the number of rejected (not properly fitted) reflections; this figure is influenced by the selection of appropriate reflections taken for a least-squares refinement. Generally, there must be linearly independent reflections numbering at least 4 to 5 times the number of crystallographic parameters to be refined, i.e. 16 to 20 at least for a monoclinic mineral with its 4 dimensions $a_{0}, b_{0}, c_{0}$ and $\beta$. Reflections with d between 1.5 and $4 \AA$ are most suitable, provided they are strong and uniquely defined. At larger angles of $2 \theta$, the ambiguity of indexing increases and the reflections are thus less suitable though their counting statistics may still be acceptable when modern, sensitive equipment is used.

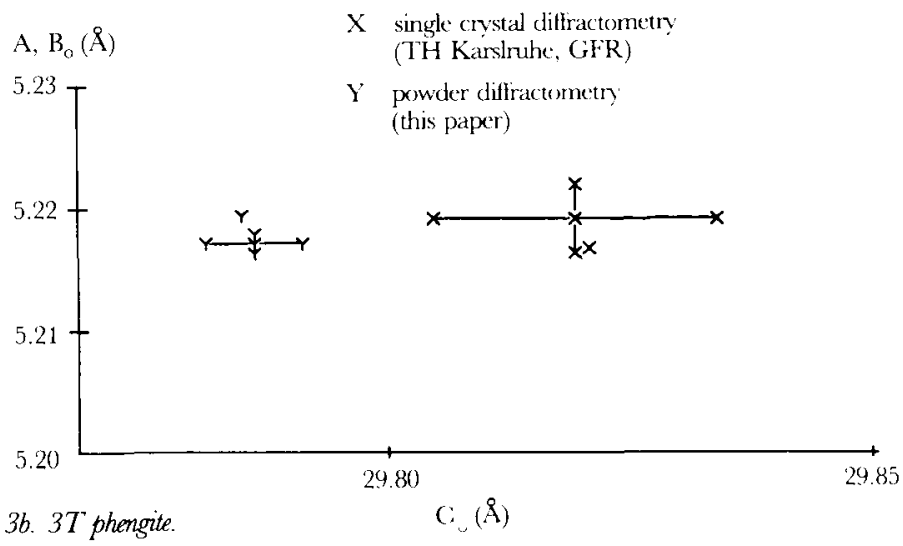




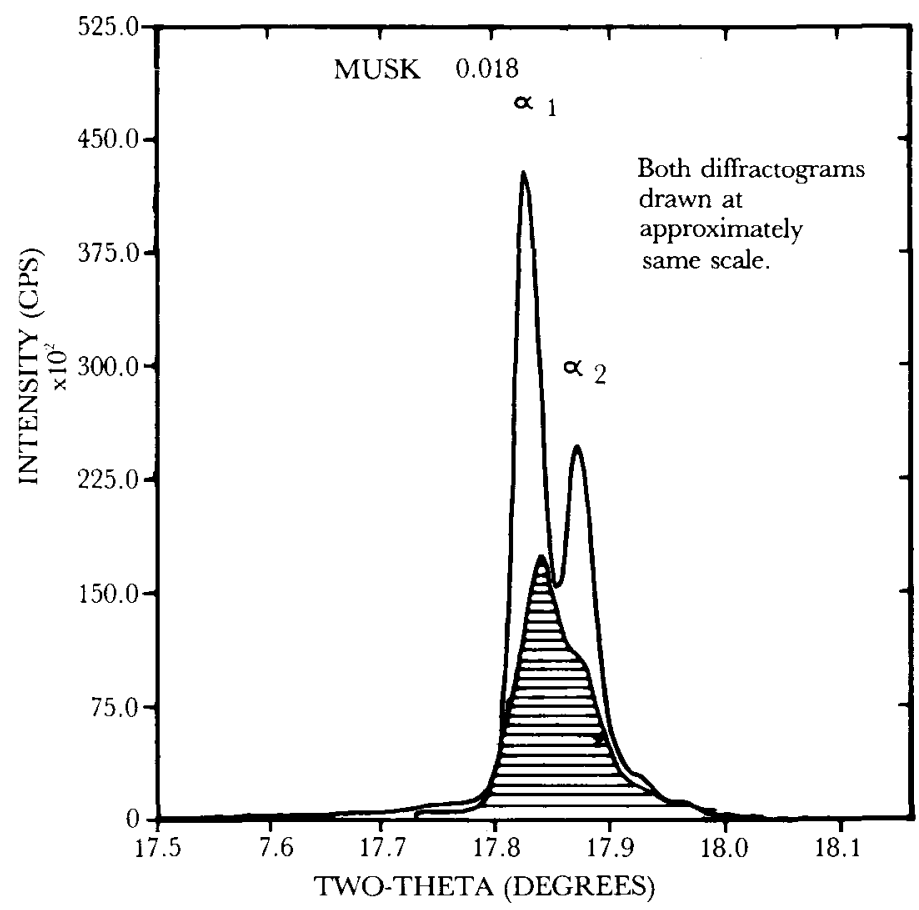

Figure 4. Companison of two XRD equipments in terms of resolution and line intensity. Same single-crystal ( $2 M_{1}$ muscovite) was measured under same excitation conditions and optimal slit configurations.

Quality of resolution and line intensity depends largely on the type of apparatus, as can be demonstrated by measuring the same specimen under comparable conditions with two different diffractometers (Figure 4). The (004) reflection of a single crystal (white $2 \mathrm{M}_{1}$ mica) displays clearly $\mathrm{K} \alpha_{1}$ and $\mathrm{K} \alpha_{2}$ when modern equipment is used, whereas only one single, relatively weak peak can be seen when an old diffractometer is used.

\section{Summary}

Data acquisition is fast and reliable in terms of precision and accuracy if a suitable specimen is measured on suitable equipment following a suitable routine. Even when measurement errors (due to sample misalignment) are properly corrected, a cell refinement with the Appleman/Evans leastsquares technique may produce ambiguous crystallographic data in case of low-symmetry minerals, like monoclinic mica. Experimental data on powder and single-crystals from the same specimens agree well

- When all unambiguous reflections are fitted (no rejections) and properly indexed.

- When the number of iterations necessary for refinement is small, $<10$.

- When the results remain the same regardless of the number of initially given (preset) Miller indices.

- When the initially given starting parameters for $a_{0}, b_{0}, c_{0}$ and $\beta$ are close to the true data (estimated acceptable difference e.g. for $b_{0}$ in the order of $0.02 \AA$ ).

One has to assume, however, that in practice incorrect refinements are not easily detected. Though the Appleman/ Evans least-squares procedure offers an interesting tool in powder diffractometry, utmost care has to be taken by critically evaluating the results. In fact, controlling refinement data is much more time-consuming than measuring specimens and processing raw data.

Finally, one may state (as one of the reviewers put it): "There is one and only one correct answer and it is the users' responsibility to locate it"?

\section{Acknowledgement}

For critical reading and useful comments the author is indebted to: Prof. M. Frey, Dr. Isenberg, Dr. H. H. Klein, Prof. H. Schwander.

\section{References}

Appleman, D. E. and Evans, H. T. Jr. (1973). Indexing and least-squares refinement of powder diffraction data. Report PB 216188, U. S. Depart. of Commerce, National Technical Information Service, 5285 Port Royal Rd., Springfield, VA 22151.

Bailey, S. W., ed. (1984) Micas. Revizwes in Mineralogy, vol. 13. Washington, DC: Min. Soc. America.

Blount, A. M. and Vassiliou, A. H. (1979). A new method of reducing preferred orientation in diffraction samples. Am. Mineral. 64, 922.

Borg, I. Y. and Smith, D. K. (1969). Calculated X-ray pouder pattems for silicate minerals. Mem. 122. Boulder, CO: Geol. Soc. Am.

Franz, G., Hinrichsen, T. and Wannemacher, E. (1977). Determination of the miscibility gap in the solid solution series paragonite-margarite, by means of infrared spectroscopy. Comtrib. Mineral. Petrol. 59, 307.

Frey, M., Hunziker, J. C., Jäger, E. and Stem, W. B. (1983). Regional distribution of white K-mica polymorphs and their phengite content in the central Alps. Contrib. Mineral. Petrol. 83, 185.

Guidotti, C. V. and Sassi, F. P. (1986). Classification and correlation of metamorphic facies series by means of muscovite $\mathrm{b}_{0}$ data from low-grade metapelites. Neues Jahrb. Mineral. 153 Abh., 363.

Naef, U., and Stem, W. B. (1982). Some critical remarks on the analysis of phengite and paragonite components in muscovite by X-ray diffractometry. Contrib. Mineral. Petrol. 79, 355.

Smith, D. K. and Leider, H. R. (1968). Low-temperature thermal expansion of $\mathrm{LiH}, \mathrm{MgO}$ and $\mathrm{CaO} . J$. Appl. Crystallogr. 1, 246.

\section{Appendix}

Analytical conditions

\section{Equipment}

Diffractometer Siemens D-500

Variable aperture, secondary monochromator.

No primary filter

Microcomputer DEC PDP 11/23+

2 hard discs $(10 \mathrm{Mb}), 1$ Winchester $(30 \mathrm{Mb})$

\section{Measurement}

Excitation Cu tube $40 \mathrm{kV} 30 \mathrm{~mA}$

Divergence $1,1^{\circ}$ entrance; $1,0.05^{\circ}$ secondary

Scan $1^{\circ} 2 \theta$ per minute, increment $0.02^{\circ} 2 \theta$

\section{Software}

Diffrac-11 Siemens

Measurement Pgms EDM, DPM

Data reduction ADR

Refinement APPLE

d-correction off-line, as described in text

\section{Sample}

50 to $200 \mathrm{mg}$ powdered mica, mechanically extracted from mother rock; or powdered megacrysts from pegmatites

Specimen holder $20 \mathrm{~mm} \phi$, spinner

Received March 9, 1987 Revised July 22, 1987 Accepted July 25, 1987 